\title{
Enhanced sugar accumulation and regulated plant hormone signalling genes contribute to cold tolerance in hypoploid Saccharum spontaneum
}

Hongli Yang ${ }^{1}$, Tianju Wang ${ }^{1,2}$, Xinghua Yu ${ }^{1,3}$, Yang Yang ${ }^{1}$, Chunfang Wang ${ }^{1}$, Qinghui Yang ${ }^{1 *}$ and Xianhong Wang ${ }^{1 *}$ (i)

\begin{abstract}
Background: Wild sugarcane Saccharum spontaneum plants vary in ploidy, which complicates the utilization of its germplasm in sugarcane breeding. Investigations on cold tolerance in relation to different ploidies in S. spontaneum may promote the exploitation of its germplasm and accelerate the improvement of sugarcane varieties.

Results: A hypoploid clone $12-23(2 n=54)$ and hyperploid clone $15-28(2 n=92)$ of S. spontaneum were analysed under cold stress from morphological, physiological, and transcriptomic perspectives. Compared with clone 15-28, clone 12-23 plants had lower plant height, leaf length, internode length, stem diameter, and leaf width; depressed stomata and prominent bristles and papillae; and thick leaves with higher bulliform cell groups and thicker adaxial epidermis. Compared with clone 15-28, clone 12-23 showed significantly lower electrical conductivity, significantly higher water content, soluble protein content, and superoxide dismutase activity, and significantly higher soluble sugar content and peroxidase activity. Under cold stress, the number of upregulated genes and downregulated genes of clone 12-23 was higher than clone 15-28, and many stress response genes and pathways were affected and enriched to varying degrees, particularly sugar and starch metabolic pathways and plant hormone signalling pathways. Under cold stress, the activity of 6-phosphate glucose trehalose synthase, trehalose phosphate phosphatase, and brassinosteroid-signalling kinase and the content of trehalose and brassinosteroids of clone 12-23 increased.
\end{abstract}

Conclusions: Compared with hyperploid clone 15-28, hypoploid clone 12-23 maintained a more robust osmotic adjustment system through sugar accumulation and hormonal regulation, which resulted in stronger cold tolerance.

Keywords: Sugarcane, Saccharum spontaneum, Hypoploidy, Cold tolerance, RNA-seq, Sugar accumulation, Hormonal change

\footnotetext{
*Correspondence: yangqinghui@163.com; x.h_wang@163.com

'Sugarcane Research Institute, Yunnan Agricultural University, Kunming 650201, Yunnan Province, PR China

Full list of author information is available at the end of the article
}

(c) The Author(s). 2020 Open Access This article is licensed under a Creative Commons Attribution 4.0 International License, which permits use, sharing, adaptation, distribution and reproduction in any medium or format, as long as you give appropriate credit to the original author(s) and the source, provide a link to the Creative Commons licence, and indicate if changes were made. The images or other third party material in this article are included in the article's Creative Commons licence, unless indicated otherwise in a credit line to the material. If material is not included in the article's Creative Commons licence and your intended use is not permitted by statutory regulation or exceeds the permitted use, you will need to obtain permission directly from the copyright holder. To view a copy of this licence, visit http://creativecommons.org/licenses/by/4.0/. The Creative Commons Public Domain Dedication waiver (http://creativecommons.org/publicdomain/zero/1.0/) applies to the data made available in this article, unless otherwise stated in a credit line to the data. 


\section{Background}

Sugarcane (Saccharum spp.) is an important sugar and energy crop, contributing $80 \%$ of the world's sugar production and $40 \%$ of that of ethanol [1]. The high sugar content genes of modern sugarcane varieties derived from tropical species (S. officinarum), however, are poorly resistant to biotic and abiotic stresses [2]. S. spontaneum belongs to the perennial herb of the family Gramineae and genus Saccharum, has both asexual and sexual reproductive abilities $[3,4]$, can grow in a variety of environments, such as drought, cold and high salt conditions [5], has varied phenotypes and strong adaptability, can provide rich genetic diversity for sugarcane breeding, and is the main source of desirable genes resistant to pests, diseases, cold, and drought $[6,7]$.

Polyploidization is a major driver of speciation [8]. Polyploidy is the characteristic of most important crops [9], such as bamboo [8], rice [10], citrus [11], wheat [11], canola [12], cotton [13] and potato [13]. According to reported estimates, $30-35 \%$ of known species and nearly $75 \%$ of Gramineae plants are polyploids [14]. Polyploids have a wider range of tolerability profiles. S. spontaneum has a basic chromosome number $\mathrm{x}=8$ with a ploidy level of 5 to 16 [15], which is a typical polyploid plant, including euploids or aneuploids with chromosome numbers ranging from $2 \mathrm{n}=40$ to 128 [16]. Moreover, polyploid S. spontaneum is capable of adapting to different environments [17], indicating that this species can be used in sugarcane breeding. Nevertheless, only the $S$. spontaneum lines Glagah $(2 \mathrm{n}=112)$, Indian $(2 \mathrm{n}=64)$, and Yacheng $(2 \mathrm{n}=64,80)$ have been successfully exploited to date $[18,19]$.

Low temperature is the most important environmental factor limiting the productivity and geographical distribution of plants around the world [20]. Climate change exacerbates the adverse effects of low temperature stress and leads to an increase in the frequency of extreme weather [21]. Sugarcane originated in the tropics. With increasing consumer demand, its planting belt has gradually expanded to the subtropical region [22]. However, subtropical winter has adversely affected the cultivation of sugarcane. The response of different sugarcane varieties significantly differs under cold stress [23] and involves differentially expressed genes [5], and miRNAs also play an important role in the cold tolerance of sugarcane [24]. Under cold stress, numerous upregulated genes were identified in S. spontaneum [25], and carbohydrate metabolism was determined to be the most significantly enriched functional pathway [26]. In-depth studies on the cold tolerance of S. spontaneum at the transcriptomic level are thus essential to its improved cold tolerance in this economically significant sugarcane species.

The Sugarcane Research Institute of Yunnan Agricultural University located in Kunming city, Yunnan
Province, China has collected and preserved nearly 600 clones of $S$. spontaneum germplasm resources since 1985 and has identified 10 types of ploidies, namely, $2 \mathrm{n}=40,48,54,60,64,78,80,88,92$, and 96 [27]. Physiological and biochemical analyses have shown that the tolerance of different ploidies of S. spontaneum to low temperature varied, with the hypoploid clone 12-23 $(2 n=54)$ being highly cold-resistant, whereas the hyperploid clone $15-28(2 \mathrm{n}=92)$ was typically cold-sensitive (Additional file 1: Supplement). The two clones were used in this study to explore the cold resistance of different ploidies of S. spontaneum in terms of morphological, physiological, and molecular characteristics, which may guide sugarcane breeding in generating new varieties with improved cold resistance.

\section{Results}

Morphological and microscopic comparison of two clones The morphological characteristics of clones 12-23 and 15-28 showed significant differences under the growth conditions of the greenhouse. Compared with clone 1528 plants, clone 12-23 plants were relatively shorter (Fig. 1a), and leaf blades were narrower (Fig. 1b). The morphological characteristics of the two clones were further measured at the maturity stage. The five morphological characteristics, including plant height, leaf length, internode length, stem diameter, and leaf width, of clone 12-23 were smaller than those of clone 15-28 (Fig. 1c). In addition, leaf anatomical analysis showed that the stomata of the 12-23 clone were depressed, and the bristles and papillae were prominent (Fig. 1d and d'), whereas the stomata of clone 15-28 were not depressed, and their bristles and papillae were fewer in number and not prominent (Fig. 1e and e'). The comparison of transverse sections of the leaves of the two clones showed that the leaf thickness of clone 12-23 was considerably larger than that of clone 15-28 (Fig. 1f and g); the bulliform cell groups of clone 12-23 was higher than that of the clone 15-28 (Fig. 1f' and g'); and the adaxial epidermal thickness of clone $12-23$ is greater than that of clone 15-28 (Fig. 1f" and g").

\section{Difference in physiological indexes between the two clones after cold stress}

After cold stress, the physiological indexes, including plasma permeability, leaf water content, soluble protein content, soluble sugar content, superoxide dismutase activity, and peroxidase activity were significantly different between clones 12-3 and 15-28 (Fig. 2). The electrical conductivity of clone 12-23 was significantly lower than that of clone 15-28 (Fig. 2a). The relative water content, soluble sugar content, soluble protein content, superoxide dismutase activity, and peroxidase activity of clone 12-23 were higher than those of clone $15-28$, of which 


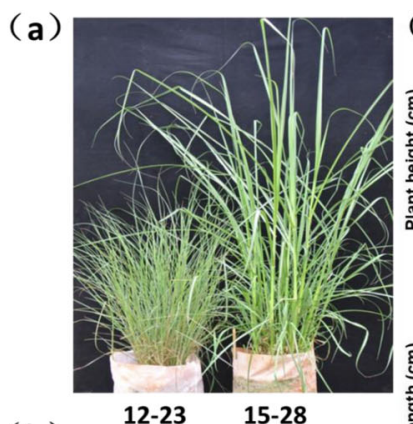

(b)

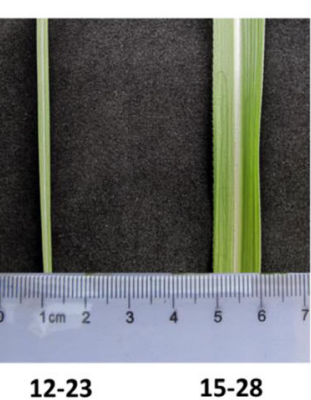

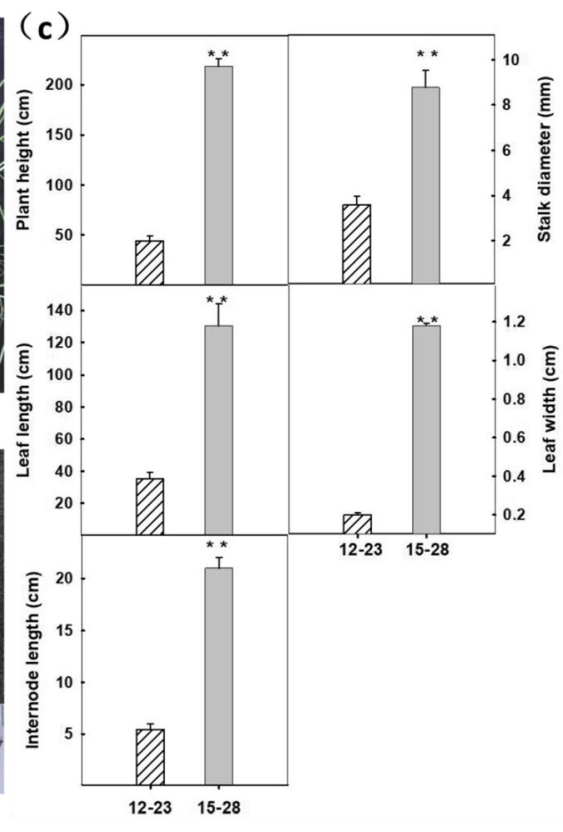
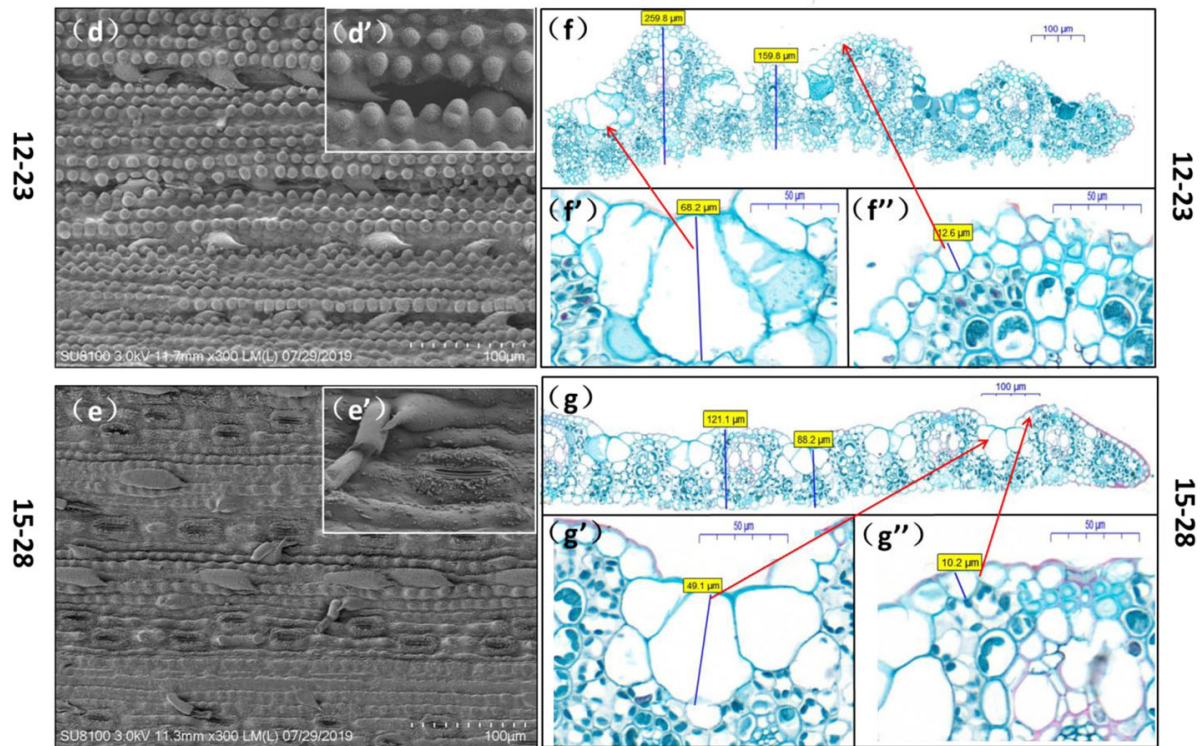

Fig. 1 Morphological features of the Saccharum spontaneum clones 12-23 and 15-28. a Plants cultivated for four months. b Leaves of plants cultivated for four months. c Plant height, leaf length, internode length, stem diameter, and leaf width of plants at the maturity stage (** indicates $p<0.01)$. Scanning electron micrograph of stomata of (d) $\left(\mathbf{d}^{\prime}\right)$ clone 12-23 and (e) (e') clone 15-28. Thickness of leaf blade, bulliform cells, and adaxial epidermis of $(\mathbf{f})\left(\mathbf{f}^{\prime}\right)\left(\mathbf{f}^{\prime \prime}\right)$ clone $12-23$ and $(\mathbf{g})\left(\mathbf{g}^{\prime}\right)\left(\mathbf{g}^{\prime \prime}\right)$ clone 15-28

the difference in relative water content, soluble protein content, and superoxide activity between clones reached significant levels $(P<0.05)$, and the difference in soluble sugar content and peroxidase activity between clones was also significant $(P<0.01)$ (Figs. $2 \mathrm{~b}-\mathrm{f})$.

\section{Differences in Transcriptomic data of clones 12-23 and 15-28 after cold stress}

Transcription is the first step in gene expression, and it is also a key step in expression regulation. Therefore, transcriptome data analysis of the two ploidy S. spontaneum was performed to further understand the cold tolerance mechanism of the hypoploid clone $12-23$ at the molecular level. Transcriptome data of the clone 12-23 low temperature stress treatment group (LL), clone 1528 low temperature stress treatment group (HL), clone 12-23 control group (LC) and clone 15-28 control group (HC) were compared. After HiSeq2500 highthroughput sequencing, clean reads were obtained for the four libraries (LL, LC, HL, and HC): 55300316, 60, 

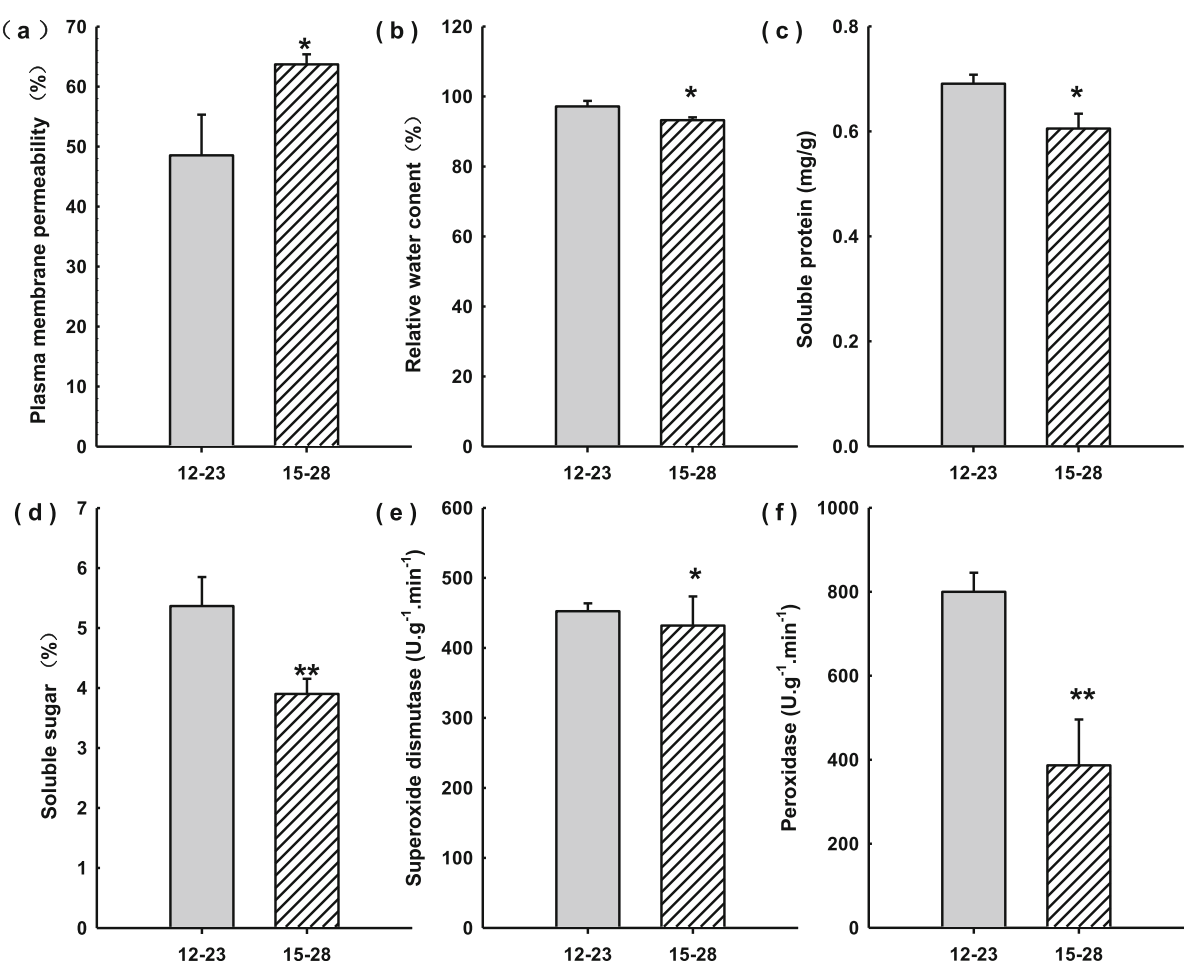

Fig. 2 Differences in various physiological characteristics between clones 12-23 and 15-28 after low temperature stress. a Cell membrane permeability. $\mathbf{b}$ Relative water content of the leaves. $\mathbf{c}$ Soluble protein content. $\mathbf{d}$ Soluble sugar content. e Superoxide dismutase content. $\mathbf{f}$ Peroxidase content. (* indicates $p<0.05{ }^{* *}$ indicates $p<0.01$ )

412,261, 58,497,202, and 45,591,750 (Additional file 2: Table S1). The data were used in the database for NR (non-redundant protein sequences), Swiss-Prot (a manually annotated and reviewed protein sequence database), Pfam (Protein family), KOG (Eukaryotic Orthologous Groups) (e-value<0.00001) comparison, and 86,275 genes were annotated. Among these genes, the most annotated genes in the $\mathrm{Nt}$ database were 75,297 (66.41\%) (Additional file 3: Table S2).

Transcriptome data of clones 12-23 included the low temperature stress group (LL) and control group (LC), and transcriptome data of clones 15-28 included the low temperature stress group (HL) and control group (HC). The transcriptomic data of clones $12-23$ and 15-28 were analysed in four pairwise comparisons: LL vs. HL, LC vs. HC, LL vs. LC and $\mathrm{HL}$ vs. HC. The total number of differentially expressed genes (DEGs) in LL vs. LC was 40,916, of which 26,417 genes were upregulated and 14,499 genes were downregulated. The total number of DEGs in $\mathrm{HL}$ vs. $\mathrm{HC}$ was 34,087 , of which 23,377 genes were upregulated and 10,710 genes were downregulated, indicating that the cold-tolerant clone $12-23$ had more up- and downregulated genes than the cold-sensitive clone 15-28. In the LL vs. HL comparison, the total number of DEGs was 31,837 , of which 14,094 genes were upregulated and 17,743 were downregulated (Fig. 3a). Further analysis showed that among the commonly shared DEGs in the four pairwise comparisons, 539 genes were upregulated, and 582 genes were downregulated (Fig. 3b).

The DEGs identified from the LL vs. HL pairwise comparison were used in Kyoto Encyclopedia of Genes and Genomes (KEGG) pathway enrichment analysis, and 20 major pathways were obtained, of which, the DEGs were primarily enriched in three pathways, namely, starch and sugar metabolism, phenylpropanoid biosynthesis, and glycolysis/gluconeogenesis, and the most significant pathways were the sugar and starch metabolic pathways (Fig. 4a). Moreover, among the pathways obtained through KEGG enrichment analysis of the DEGs identified from LL vs. HL, the plant hormone signal transduction pathway had the highest number of DEGs (Fig. 4b). Further comparison of the $P$-value of the pathways obtained through KEGG enrichment analysis of DEGs identified from the pairwise comparisons of LL vs. LC and HL vs. HC showed that the plant hormone signalling transduction pathway was the most significantly enriched (Fig. 4c). Based on these results, we focused our analysis on the sugar and starch metabolic and plant hormone signalling pathways. 


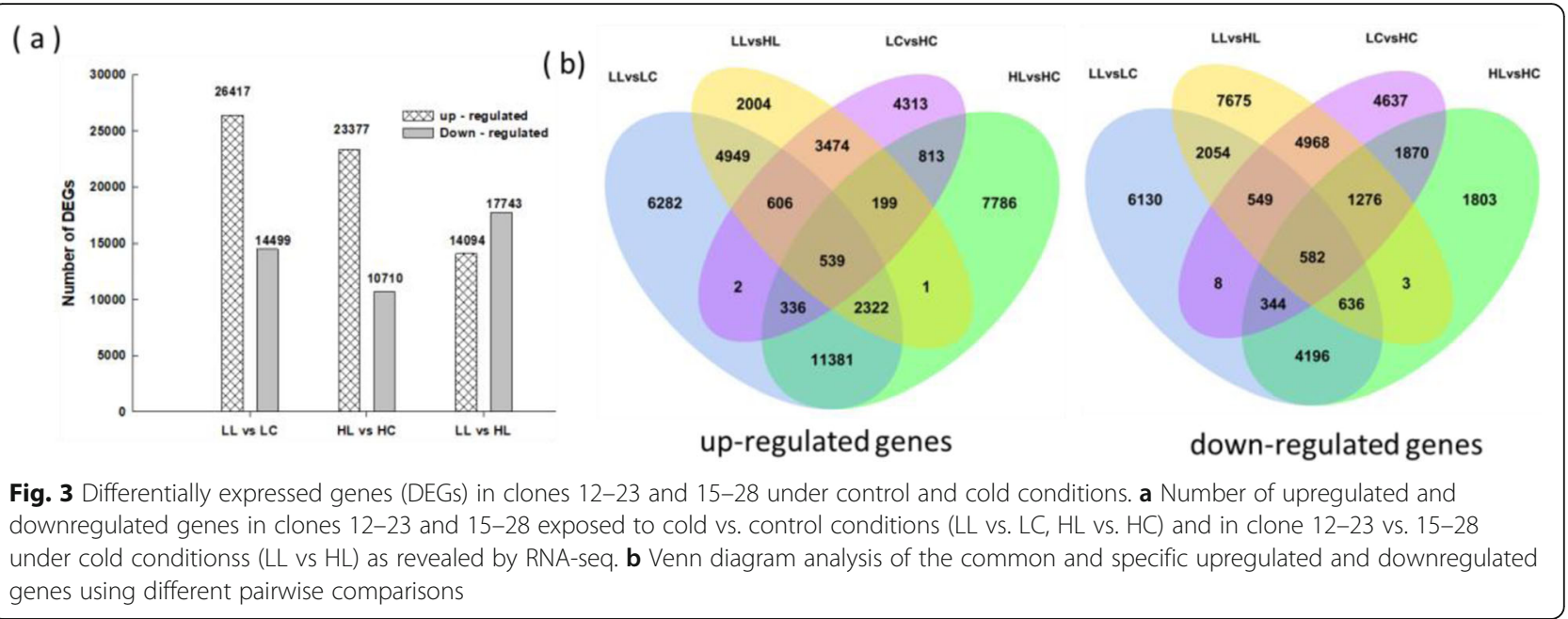

\section{Enhanced sugar accumulation contributes to cold} tolerance in S. spontaneum

KEGG pathway enrichment analysis of DEGs from LL vs. HL showed that the sugar and starch metabolic pathways were most significantly enriched. The DEGs in the sugar and starch metabolic pathways were further analysed by means of ORFfinger alignment and functional annotation ( $\mathrm{KO}$ and $\mathrm{GO}$ ), and then the FPKM values of the screened genes were compared. We found that the FPKM values of the 6-phosphate glucose trehalose synthase (TPS) and trehalose phosphate phosphatase (TPP) genes controlling trehalose synthesis were the highest (Fig. 5a). The differential expression of TPS1, TPS2, and TPP was validated by RT-qPCR using $18 S$ rRNA and $G A P D H$ genes as internal references. The results showed that the differential fold change in the expression of genes detected by RT-qPCR was different from that revealed by RNA-seq, but the trends of gene expression presented by the two methods were largely the same (Fig. 5b) (Additional file 4: Figure S1). Furthermore, we determined the change in trehalose content in clones 12-23 and 15-28 under cold stress and control conditions. Under control conditions, the two clones did not show significant differences in trehalose content. After cold stress, trehalose content increased in both clones, although the trehalose content of clone 12-23 was significantly higher than that of clone 15-28 (Fig. 5c).

\section{Regulated plant hormone Signalling genes contribute to cold tolerance in S. spontaneum}

For the DEGs obtained from RNA-seq analysis, analysis of FPKM value (expression level), $\log _{2}$ (fold change), ORFfinger alignment, and functional annotation (KO, $\mathrm{GO}$ ) revealed that the $\log _{2}$ (fold change) value of the brassinosteroid signal kinase (BSK) was the highest (Fig. 6a), and for both clones 12-23 and 15-28, the FPKM values increased after cold stress (Fig. 6b). The expression of the $B S K$ gene was verified by RT-qPCR using $18 S$ rRNA and GAPDH genes as internal references. The differential fold change in gene expression using RT-qPCR differed from that shown by RNA-seq, but the trend in gene expression presented by the two methods was largely the same (Fig. 6b) (Additional file 5: Figure S2). We further determined the change in brassinosteroid content of clones 12-23 and 15-28 subjected to cold stress. The difference in brassinosteroid content between the two clones was significant under control conditions. After cold treatment, the brassinosteroid content increased in both clones, and its content was significantly higher in clone $12-23$ than in clone $15-28$ (Fig. 6c).

\section{Discussion}

Morphological and physiological characteristics of lowploidy and high-ploidy of S. spontaneum vary with cold tolerance

A previous study on $S$. spontaneum indicated that phenotypes differed with ploidy. Low-ploidy plants tend to be short and form thick leaves [28]. Plant leaves are the main organ responding to environmental affecters, and their anatomical features have an important impact on their adaptability to specific environments [29]. As a channel for the leaves to absorb $\mathrm{CO}_{2}$ and dissipate moisture, stomata regulate and control the water use efficiency of plants. Stomata are also in the joint point of primary productivity of terrestrial ecosystems with water transpiration and can be used as an indicator of the strength of plant resistance [30]. Depressed stomata may reduce moisture evaporation and are a characteristic of stress-resistant plants [31]. Bulliform cells are unique leaf structures of gramineous plants. Plants with a high number and/or large volume of bulliform cells tend to have greater stress resistance [32]. Water storage and supply of a plant have a close relationship with leaf 
(a)

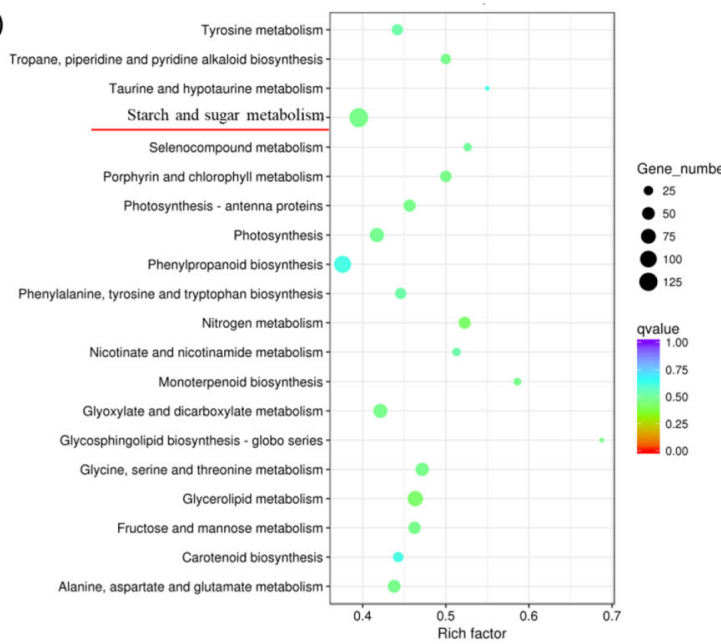

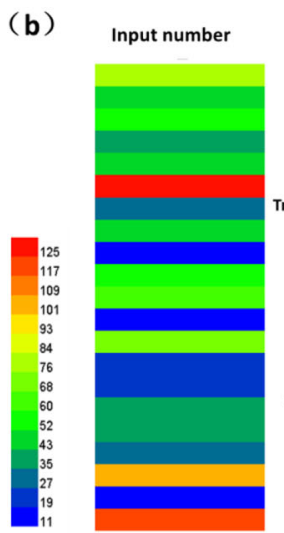

(c)
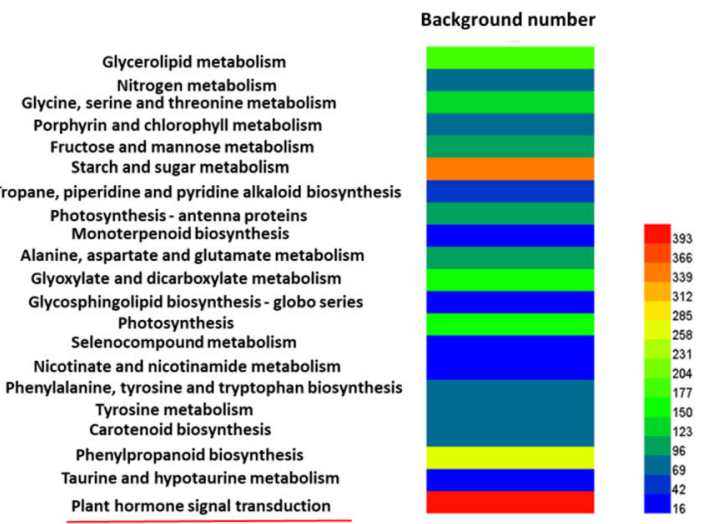

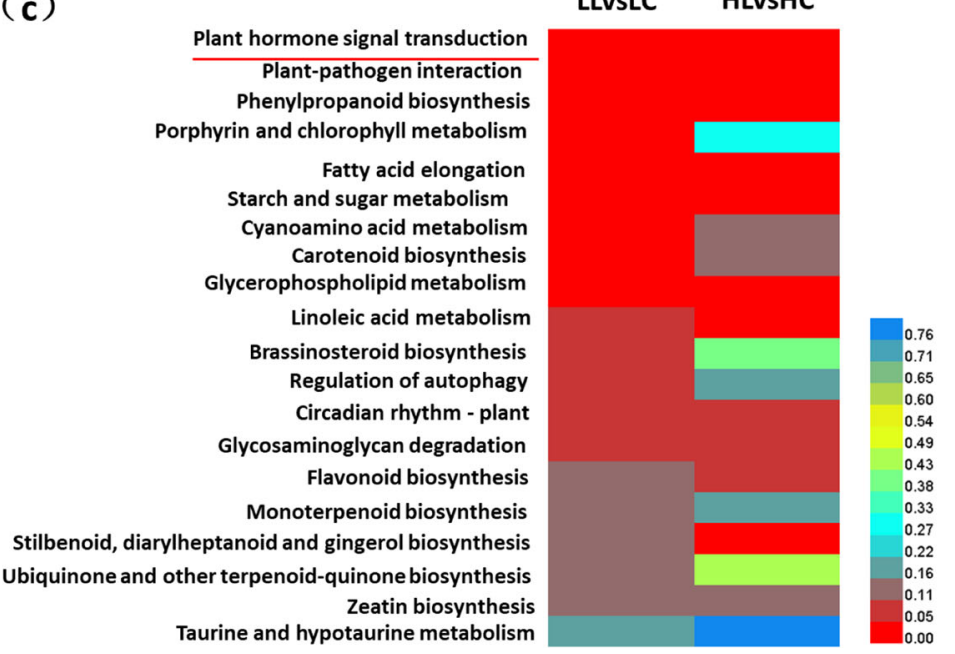

Fig. 4 Kyoto Encyclopedia of Genes and Genomes (KEGG) pathway analysis of differentially expressed genes (DEGs). a The top 20 enriched pathways of the DEGs between clones 12-23 and clone 15-28 under cold conditions (LL Vs. HL). The X-axis indicates the enrichment factor on a scale from 0 to 0.7 . The dot colour and size indicate the q-value and gene number as shown on the right. $\mathbf{b}$ Heat map of input number and background number of the KEGG enriched pathways of the DEGs of clones under cold conditions (LL vs. HL). The left colour panels display the input number of each term from 11 to 125, and the right colour panels display the background number of each term from 16 to 393 . c Heat map of the KEGG enriched pathways of the DEGs of the pairwise comparisons LL vs. LC and HL vs. HC. Colour panels display the $P$-values of each term from 0 to 0.76 


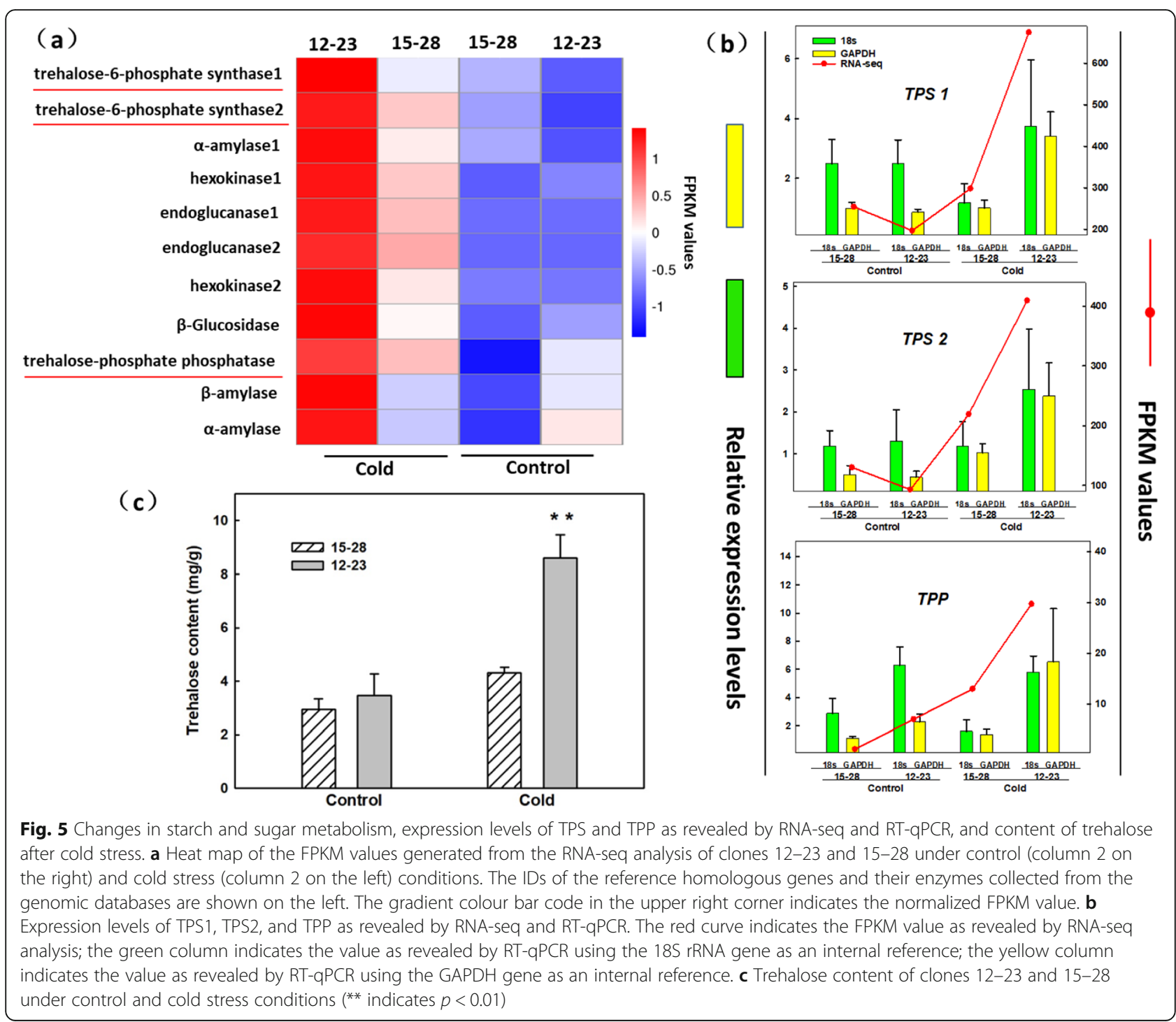

thickness [33]. The results of this study indicated that the stomata of clone 12-23 were depressed, and the bristles and papillae were prominent, whereas clone 1528 did not exhibit such characteristics. In addition, clone 12-23 showed taller bulliform cells and thicker adaxial epidermis than that of clone 15-28. Thus, based on the anatomical structure of the leaves, clone $12-23$ possesses cold-tolerant characteristics.

When plants are exposed to low temperature, the first change that occurs is cell membrane permeability. The membrane is a protective barrier between plant cells and the external environment. The membrane can not only receive and transmit environmental signals, but also respond to environmental stress. Low temperature may damage cell membranes, thereby increasing their permeability. The relative permeability of the cell plasma membrane is positively correlated with the degree of cell membrane damage [23]; therefore, the value of the membrane permeability is negatively correlated with cold tolerance. Soluble proteins and soluble sugars are important osmoregulation substances in plants. The content of these proteins and sugars increases at low temperature, acting as anti-dehydrating agents to reduce the water potential of cells and enhance the water holding capability, thereby reducing plant damage [34]. Under abiotic stress, plants may accumulate reactive oxygen species (ROS). If not removed, excessive ROS can cause damage to nucleic acids, proteins, and lipids [35]. Plants have evolved an antioxidant defence system, of which superoxide dismutase and peroxidase are the strongest antioxidant enzymes [21] and are important physiological indexes for cold tolerance detection. The results of our study indicated that the physiological indexes of the two clones were significantly different under cold stress. The electrical conductivity of clone 12-23 was lower than that of clone 15-28. The relative water 


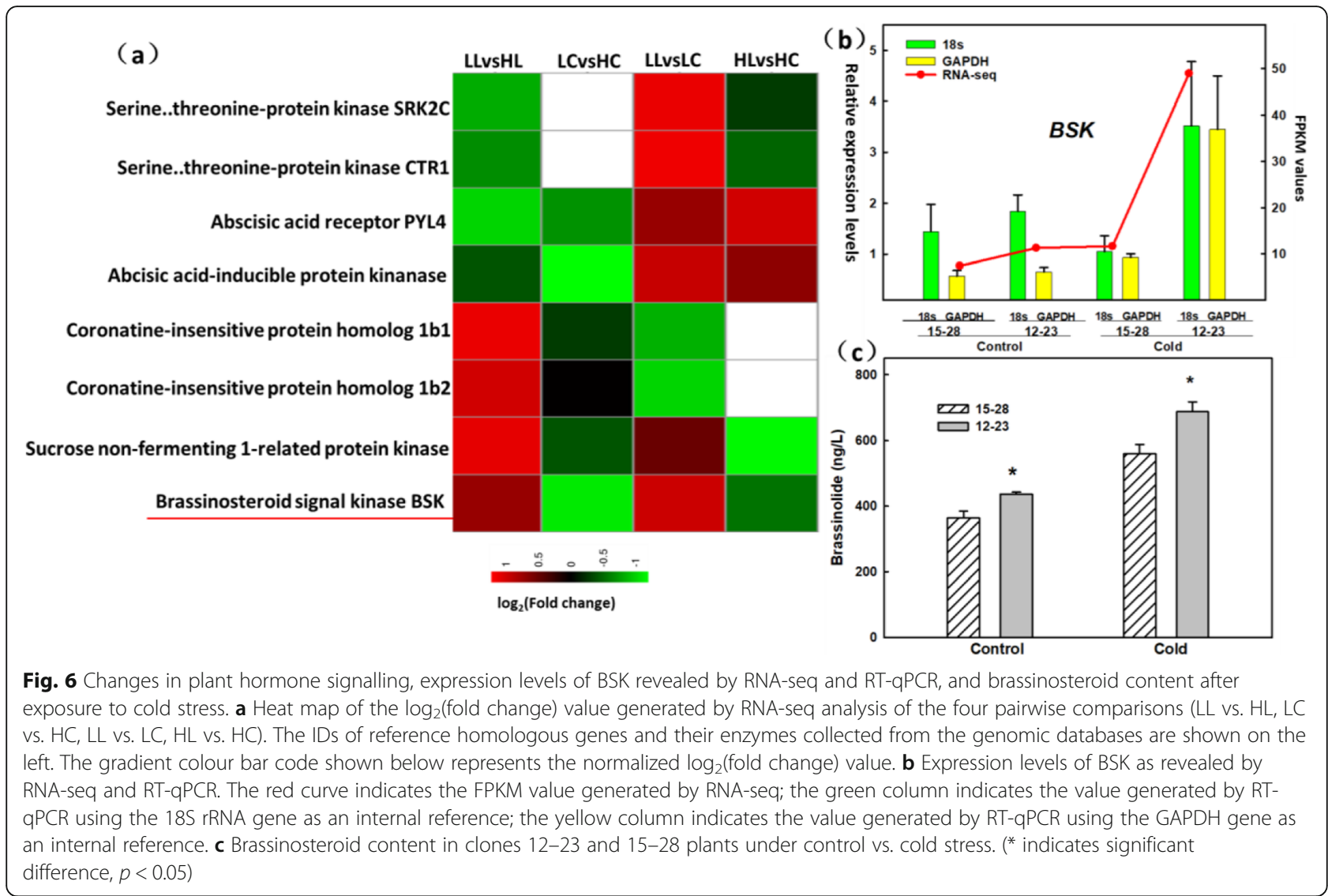

content, soluble sugar content, soluble protein content, superoxide dismutase activity, and peroxidase activity of clone 12-23 were greater than those of clone 15-28. These results indicate that clone $12-23$ is more tolerant to cold.

\section{Transcriptomic difference between cold-resistant and} cold-sensitive clones of $S$. spontaneum in response to cold stress

Transcriptional regulation is an important regulatory mechanism of the transcriptome in response to abiotic stress in plants [36]. Studies have shown that coldtolerant S. spontaneum produces more DEGs than other sugarcane varieties under cold stress [5]. In this study, the transcriptomic analysis that clone 12-23 revealed changes in gene expression under cold stress, which might involve more metabolic pathways and regulatory mechanisms to adapt to the cold environment.

Multiple genes interact with each other in different pathways and play roles in the abiotic stress response. KEGG is a database that integrates genomic, chemical, and systemic functional information [37]. Under certain circumstances, soluble sugar and starch can be converted into each other. As an intermediate or end product of metabolism, sugar can regulate many physiological processes such as plant growth, development, and formation of stress resistance, indicating that the sugar and starch metabolic pathways play an important role in the enhancement of cold tolerance in plants [38]. In addition, plant hormones can regulate the growth and development of plants, which include auxins, cytokines, gibberellins, abscisic acid, ethylene, and brassinosteroids. Hormones are involved in the normal development of plants and play an important role in stress responses [39]. In this study, we found that among all the pathways revealed through KEGG enrichment analysis of the DEGs from the LL vs. HL pairwise comparison, the plant hormone signal transduction pathway showed the highest enrichment. Comparison of $P$-values of the KEGG enriched pathways of DEGs from clones $12-23$ and 15-28 under cold stress indicated that for the pairwise comparisons of LL vs. LC and HL vs. HC, the plant hormone signalling transduction pathway was the most significantly enriched pathway by KEGG.

Increase in Trehalose content and changes in plant hormone expression significantly enhances cold tolerance in low-ploidy S. spontaneum

Several soluble sugars, such as sucrose, glucose, fructose, ribose, and trehalose, often accumulate under low temperature stress [11]. These sugars can scavenge free 
radicals and indirectly induce protein synthesis, thereby improving the cold tolerance of plants [25]. The results of this study indicate that the 6-phosphate glucose trehalose synthase (TPS) and the trehalose phosphate phosphatase $(T P P)$ that synthesize trehalose are significantly different when comparing the two materials (LL vs. HL) under low temperature stress. Trehalose is a safe, nonreducing disaccharide that is found in fungi, bacteria and insects [40]. Trehalose plays a role in protecting biomacromolecules, such as membrane proteins, and hence is significant to organism survival [41]. Under adverse conditions such as drought, cold, and high salt, plants can produce trehalose to help resist these external influences. An earlier study showed that trehalose could be detected only in "resurrected plants" that have notably strong drought tolerance [42]. In 1969, trehalose was detected in sugarcane seedlings [43]. TPS and TPP are the most important enzymes for the synthesis of trehalose [44]. 6-Phosphate glucose trehalose synthase (TPS) catalyses the formation of 6-phosphate trehalose (T6P) from UDP-glucose and 6-phosphate glucose, and then T6P is catalysed by trehalose phosphate phosphatase $(T P P)$ to produce trehalose. TPS and TPP are also called OtsA and $O t s B$, respectively [41]. Studies have shown that tobacco with the trehalose synthesis gene showed strong drought tolerance [45], and the transgenic rice plants overexpressing these genes exhibited significantly enhanced tolerance to cold, drought, and high salt levels [46]. Interestingly, we found that under cold stress, the trehalose content of clone 12-23 was higher than that of clone 15-28, suggesting that TPS and TPP play an important role in the enhancement of cold tolerance of hypoploid S. spontaneum.

Studies have shown that ABA signalling activates the expression of plant cold resistance genes mediated by ABREs (ABA responsive element binding protein) to enhance cold resistance [47]. ABA signal transduction is largely dependent on the protein phosphatase $P P 2 C$ [48]. The results of this study showed that $P P 2 C$ related to ABA synthesis was significantly higher than that of the control group under low temperature stress, indicating that ABA plays an important role in resistance to low temperature stress. The comparison between lowploidy clones 12-23 and high-ploidy clones 15-28 under low temperature stress indicated that brassinolactone was the major hormone variable, that is, by comparing $\log _{2}$ (fold changes) and FPKM values, we found that BSKs were the most frequent signals involved in responses to cold stress. Recently, brassinosteroids (BRs), steroid hormones other than the five classic plant hormones were discovered [49]. Several studies have shown that it can control photosynthesis, influence carbohydrate metabolism, defend plants from environmental affect [50], and confer resistance to biotic and abiotic stresses [51, 52].
BRs can activate the oxidase protection system in plants, thereby eliminating the excessive harmful free radicals derived from stresses, thereby improving the stress resistance of plants [53]. Brassinosteroid signal kinases (BSKs) are the downstream regulatory elements of BRs that bind to BRI1, which is located on the membrane and is activated as a substrate of BRI1 downstream of BR, thereby transmitting BR signals downstream, mediating the BRinduced increase in the activity of antioxidant protective enzymes [54]. It has been reported that BSK5 plays an important role in drought tolerance [55]. RT-qPCR analysis revealed that the expression profile of $B S K s$ coincided with the results obtained through RNA-seq. Furthermore, the BR content in clone 12-23 was significantly higher than that in clone 15-28 under cold stress, further indicating that the BSK gene plays an important role in cold tolerance in hypoploid S. spontaneum.

\section{Potential applications of Hypoploid S. spontaneum in sugarcane cold resistance breeding}

In traditional polyploidy studies, the prevailing view is that polyploids have greater stress resistance [11]. Peer et al. [56] discussed the significance of polyploidy in evolution and noted that the concept of increased ploidy leading to a wide range of tolerance levels remains controversial. The results of our study indicated that hypoploid S. spontaneum plants are more resistant to cold than hyperploid plants. Liu et al. [28] showed that $2 n=64$ S. spontaneum from Yunnan has better quality and yield than the other four S. spontaneum ploidies $(2 \mathrm{n}=64,72,80,96)$. Zhang et al. [2] identified $80 \%$ of disease resistance genes on the rearranged chromosomes of haploid material $(2 n=32)$ derived from the pollen culture of hypoploid S. spontaneum, indicating that the reduction in chromosome number facilitated the retention of disease resistance genes. Yu et al. [27] showed that ploidy evolution and the distribution of S. spontaneum may be correlated with habitat features, such as latitude and altitude. The systematic evolution of different ploidies of S. spontaneum and their stress tolerance thus merit further investigation.

Sugarcane breeding plays an important role in improving sugarcane yield and resistance. However, due to insufficient development and rational utilization of germplasm resources, as well as a long breeding cycle [7], sugarcane breeding is relatively slower than other crops. S. spontaneum is the most important wild sugarcane germplasm [57] and is a typical polyploid resource that is rich in resistance-related genes [58]. An in-depth study of the resistance of different ploidies of $S$. spontaneum and their rational use are thus of profound significance for accelerating sugarcane breeding. In our study, morphological, physiological, and transcriptomic analyses showed that the hypoploid $S$. spontaneum clone $12-23(2 n=54)$ possesses strong cold tolerance and can 
be utilized to generate novel cold-tolerant sugarcane varieties. The results of our study improve our understanding of the morphological characteristics and molecular mechanisms of cold tolerance in hypoploid S. spontaneum.

\section{Conclusions}

In this study, the cold tolerance of hypoploid and hyperploid clones of S. spontaneum was analysed through morphological, physiological, and transcriptomic data. Compared with the hyperploid clones 15-28, the hypoploid clones 12-23 exhibited different morphological and physiological characteristics, and exhibited significant tolerance to cold. The transcriptomic data showed that low temperature stress altered the expression of many stress response genes and pathways in clone 1223, particularly sugar and starch metabolic pathways and the plant hormone signalling pathway. Our findings suggest that under low temperature conditions, the hypoploid S. spontaneum clone maintains a more robust osmotic regulatory system than the hyperploid clone through sugar accumulation and hormonal changes, and thus has a stronger resistance to low temperatures. The results of this study help to elucidate the morphological characteristics and molecular mechanisms underlying the strong cold tolerance of hypoploid S. spontaneum.

\section{Methods}

\section{Plant materials}

S. spontaneum cold-resistant clone $12-23(2 \mathrm{n}=54$, collected from Tibet, China) and the cold-sensitive clone 15$28(2 n=92$, collected from Burma) were harvested from the resource conservation room of Sugarcane Research Institute, Yunnan Agricultural University in Kunming city, Yunnan Province, China. The ploidy identification of two clones is shown in Additional file 6: Figure S3.

\section{Morphometric measurement}

At the mature stage, the agronomic traits of the clones were investigated. Five plants from each clone were selected for measuring plant height, stem diameter, leaf length, leaf width, and internode length (Additional file 7: Table S3). Assessment of traits was based on the Technical Code for Evaluating Crop Germplasm ResourcesSugarcane (NY/T1488-2007) [59].

\section{Microscopic observation}

At maturity, three disease-free plants with the same growth status were selected from the two clones. Three leaves in the middle of each plant were collected for paraffin sectioning. We fixed the fresh tissue with fixative for more than $24 \mathrm{~h}$, removed the tissue from the fixative and placed it into the dehydrator (Wuhan Junjie Electronics Co., Ltd., JJ-12 J) for dehydration with gradient alcohol. Dehydrated samples were embedded in an embedding machine (Wuhan Junjie Electronics Co., Ltd., JB-P5). The embedded paraffin sections were placed on a microtome (Shanghai Leica Instrument Co., Ltd., RM2016) for sectioning, and each section was $3 \mu \mathrm{m}$ thick. Finally, dewaxing, safranin $\mathrm{O}-$ fast green staining, and photography were conducted, and the crosssectional anatomy of the leaves was assessed by Caseviewer 2.0 software [11]. The stomata were observed using a scanning electron microscope. First, we limited mechanical damage when taking the material, quickly taking each sample within 1-3 min. The tissue block did not exceed $3 \mathrm{~mm}^{2}$, and was quickly placed in the electron microscope fixing solution (Servicebio, G1102) for $2 \mathrm{~h}$ at room temperature. Then the samples were transferred to a $4{ }^{\circ} \mathrm{C}$ refrigerator and stored in the dark. Then, the leaves were removed and dehydrated with ethanol. After dehydration, the sample was placed in a critical point dryer (Quorum, K850) for drying. Finally, the sample was placed on the double-sided adhesive tape of the conductive carbon film and placed on the sample table of the ion sputtering apparatus (IXRF, MSP-2S). The gold was sprayed on the sample stage of the ion sputtering apparatus (HITACHI, SU8010) [11].

\section{Physiological measurements}

Seedlings (stage with 5-6 leaves) of S. spontaneum were subjected to cold stress in an incubator. The growth conditions were as follows: temperature, $3{ }^{\circ} \mathrm{C}$; light intensity, $250-300 \mu \mathrm{mol} \cdot \mathrm{m}^{-2} \cdot \mathrm{s}^{-1}$; photoperiod, $12 \mathrm{~h}$; and relative humidity $60-70 \%$. After 3 days of cold stress treatment [5], the leaves of the seedlings from the control and low temperature stress treatments were collected, and 3 replicates of sampling were used per treatment. The +1 leaves (top visible dewlap leaf) of the same size and phenotype were collected as the test material. Cell membrane permeability was measured as described by Huang et al. (2015) using a conductivity meter (DDSJ-308F, Leici, China) [60]. The leaf relative water content index was determined by selecting the +1 leaf and recording its fresh weight (labelled as $\mathrm{W}_{\mathrm{f}}$ ) according to the method described by Cia et al. [61]. Then, each leaf was soaked in water for 5-6h, dried, and weighed. Then each leaf was soaked in water for $1 \mathrm{~h}$, dried, and weighed again. This operation was repeated until the saturated weight of the sample was stable, and the saturated fresh weight was recorded (labelled $\mathrm{W}_{\mathrm{t}}$ ). Then G2 was dried in an oven to a constant weight, and the dried sample was weighed (labelled $\mathrm{W}_{\mathrm{d}}$ ). We calculated the relative water content according to the formula: $\left(\mathrm{W}_{\mathrm{f}}-\mathrm{W}_{\mathrm{d}}\right) /\left(\mathrm{W}_{\mathrm{t}}-\mathrm{W}_{\mathrm{d}}\right) \times 100 \%$. Soluble sugar content was determined using the fluorenone colorimetric method [23]. The soluble protein content was determined using the Coomassie Brilliant Blue $\mathrm{G}^{-250}$ method [23]. Superoxide dismutase, peroxidase, trehalose, and 
brassinosteroid contents were determined using a kit manufactured by Suzhou Keming Biotechnology Co., Ltd. The determination was performed in triplicate for each sample.

\section{RNA-seq analysis}

The treatment of the plant materials was the same as that in Section 1.3, which included cold stress and the control. Clone 12-23 under cold stress was labelled LL, and the control was labelled LC. Clone 15-28 under cold stress was labelled HL, and the control was labelled HC. Three biological replicates were used for each treatment. The +1 leaves (top visible dewlap leaf) of basically the same size and phenotype were used in RNA extraction and RNA-seq analysis. RNA quality was detected using a NanoDrop. Clean reads were assembled using Trinity software (version 2.0.6) [62]. The obtained transcriptomic sequences were submitted to the databases of NCBI non-redundant protein sequences (NR) and nucleotide sequences (NT), protein family (Pfam), Eukaryotic Orthologue Groups (KOG), Kyoto Encyclopedia of Genes and Genomes (KEGG), and Gene Ontology (GO) to perform BLASTX analysis to obtain the best annotation (E-value $\leq 1 \mathrm{E}-03)$. Gene expression levels were assessed using RSEMV 1.2.12 software [63]. The expression level of a single gene was calculated using the expected number of fragments per kilobase of transcript sequence per million base pairs sequenced (FPKM) formula. Differentially expressed genes (DEGs) were defined using $\log _{2} \mid$ Fold Change $\mid>1$ and FDR (false discovery rate) $<0.05$.

\section{Real-time fluorescent quantitative PCR detection}

Real-time fluorescent quantitative reverse transcription PCR (RT-qPCR) was used to quantify the relative expression levels of genes. RNA of the leaf samples described above was extracted for RT-qPCR analysis. GAPDH [64] and 18S rRNA [65, 66] genes were used as internal references. The RNA OD value was measured using an Analytik Jena ${ }^{\text {tm }}$ Scandrop $^{\text {tw }} 100$ Spectrophotometer, and the A260/A280 ratio was calculated. The TRUEscript 1st Strand cDNA synthesis kit manufactured by Aidlab was used for reverse transcription. A $20-\mu \mathrm{L}$ reaction system (Additional file 8: Table S4) was used, and each reaction was performed thrice. After amplification, a melting curve was generated, and the $2^{-\Delta \Delta \mathrm{Ct}}$ method was used to calculate the relative expression levels of genes [67]. The primers used for RT-qPCR are listed in Additional file 9: Table S5.

\section{Data analysis}

Microsoft Excel 2010 was used in the data analysis. SPSS (v17.0, IBM, New York, NY, US) was used in variance analysis [68]. SigmaPlot (v10.0, Chicago, IL, US) software was used to contruct graphs [69]. HemI (v1.0, China) software was used to prepare heat maps (http://hemi. biocuckoo.org/index.php) [70].

\section{Supplementary information}

Supplementary information accompanies this paper at https://doi.org/10. 1186/s12864-020-06917-z.

\section{Additional file 1: Supplement.}

Additional file 2: Table S1. Overview of transcriptome sequencing and de novo assembly results.

Additional file 3: Table S2. Summary of the functional annotation of assembled unigenes.

Additional file 4: Figure S1. Analysis of variance of RT-qPCR expression of TPS and TPP.

Additional file 5: Figure S2. Analysis of variance of RT-qPCR expression of BSK.

Additional file 6: Figure S3. Ploidy identification of $12-23$ clones and 15-28 clones.

Additional file 7: Table S3. Index and content of investigation on agronomic characters.

Additional file 8: Table S4. Quantitative real-time PCR.

Additional file 9: Table S5. Primers used in quantitative real-time RTqPCR validation of gene expression data as revealed by RNA-seq analysis.

\section{Abbreviations}

DEGs: Differentially expressed genes; KEGG: Kyoto Encyclopedia of Genes and Genomes; GO: Gene Ontology; KOG: Eukaryotic Orthologue Groups;

NT: Nucleotide sequences; NR: Nonredundant protein sequences;

Pfam: Protein family; FPKM: Ragments per kilobase of transcript sequence per million base pairs sequenced; FDR: False discovery rate

\section{Acknowledgements}

Not Applicable.

\section{Authors' contributions}

HLY, XHW and QHY designed the experiments; HLY, TJW, YY, CFW and XHY performed the research; HLY analysed the data and wrote the manuscript; and XHW and QHY helped to revise the manuscript. All authors read and approved the final manuscript.

\section{Funding}

This study was financially supported by the National Natural Science Foundation of China (No. 31760417 and No. 31460372). The two funders are the corresponding authors of this paper. No. 31760417: sequencing and RTqPCR analysis. No. 31460372: collection and culture of experimental materials.

\section{Availability of data and materials}

The data supporting the conclusions of this article are within the paper and its additional files. All sequencing reads are deposited in the National Center for Biotechnology Information under the BioProject number PRJNA589716 with the Sequence Read Archive (SRA) study accession SRP230310.

Ethics approval and consent to participate

Not applicable. We declare that the two S. spontaneum clones employed for the study do not require any permissions.

Consent for publication

Not applicable.

\section{Competing interests}

The authors declare that they have no competing interests.

\section{Author details}

'Sugarcane Research Institute, Yunnan Agricultural University, Kunming 650201, Yunnan Province, PR China. ${ }^{2}$ Chuxiong normal university, Chuxiong 675000, Yunnan Province, PR China. ${ }^{3}$ Wenshan Academy of Agricultural Sciences, Wenshan 663000, Yunnan Province, PR China. 
Received: 19 November 2019 Accepted: 15 July 2020 Published online: 22 July 2020

\section{References}

1. Wang JP, Roe B, Macmil S, Yu QY, Murray JE, Tang HB, Chen CX, Najer F, Graham W, Bowers J, et al. Microcollinearity between autopolyploid sugarcane and diploid sorghum genomes. BMC Genomics. 2010;11:261-78.

2. Zhang JS, Zhang XT, Tang HB, Zhang Q, Hua XT, Ma XK, Zhu F, Jones T, Zhu $X G$, Bowers J, et al. Allele-defined genome of the autopolyploid sugarcane Saccharum spontaneum L. Nat Genet. 2018;50(11):1565-73.

3. Evans DL, Joshi SV. Complete chloroplast genomes of Saccharum spontaneum, Saccharum officinarum and Miscanthus floridulus (Panicoideae: Andropogoneae) reveal the plastid view on sugarcane origins. Syst Biodivers. 2016;6(14):548-71.

4. Panje RR, Bab CN. Studies in Saccharum spontaneum distribution and geographical association of chromosome Numbers' ${ }^{1}$. Cytologia. 1960;25: 152-72.

5. Park JW, Benatti TR, Marconi T, Yu QY, Gracia NS, Mora V, Silva JAD. Cold responsive gene expression profiling of sugarcane and Saccharum spontaneum with functional analysis of a cold inducible Saccharum homolog of NOD26-like intrinsic protein to Salt and water stress. PLoS One. 2015;10:e01258102015.

6. Govindaraj P, Amalraj VA, Mohanraj K, Nair NV. Collection, characterization and phenotypic diversity of Saccharum spontaneum L. from arid and semi arid zones of northwestern India. Sugar Tech. 2014;16(1):36-43.

7. Ming R, Moore PH, Wu KK, Angelique DH, Jean CG, Tew TL. Sugarcane improvement through breeding and biotechnology. Plant Breed Rev. 2006; 27:15-118.

8. Guo ZH, Ma PF, Yang GQ, Hu JY, Liu YL, Xia EH, Zhong MC, Zhao L, Sun HL, $X u Y X$, et al. Genome sequences provide insights into the reticulate origin and unique traits of Woody bamboos. Mol Plant. 2019;12(10):1353-65.

9. Osborn TC, Pires JC, Birchler JA, Auger DL, Chen ZJ, Lee HS, Comai L, Madlung A, Doerge RW, Colot V, Martienssen RA. Understanding mechanisms of novel gene expression in polyploids. Trends Genet. 2003; 3(19):141-7.

10. Yang PM, Huang QC, Qin GY, Zhao SP, Zhou JG. Different drought-stress responses in photosynthesis and reactive oxygen metabolism between autotetraploid and diploid rice. Photosynthetica. 2014;52(2):193-202.

11. Wei TW, Wang Y, Xie ZZ, Guo DY, Chen CW, Fan QJ, Deng XD, Liu JH. Enhanced ROS scavenging and sugar accumulation contribute to drought tolerance of naturally occurring autotetraploids in Poncirus trifoliata. Plant Biotechnol J. 2019;17(7):1394-407.

12. Soltis PS, Soltis DE. Ancient WGD events as drivers of key innovations in angiosperms. Plant Biol. 2016;30:159-65.

13. Chao DY, Dilkes B, Luo HB, Douglas A, Yakubova E, Lahner B, Salt DE. Polyploids exhibit higher potassium uptake and salinity tolerance in Arabidopsis. Science. 2013;341:658-9.

14. Ha S, Moore PH, Heinz D, Kato S, Ohmido N, Fukui K. Quantitative chromosome map of the polyploid Saccharum spontaneum by multicolor fluorescence in situ hybridization and imaging methods. Plant Mol Biol. 1999;39(6):1165-73.

15. Irvine JE. Saccharum species as horticultural classes. Theor Appl Genet. 1998; 98(2):186-94.

16. Silva JAD. The importance of the wild cane Saccharum spontaneum for bioenergy genetic breeding. Sugar Tech. 2017;19(3):229-40.

17. Ha M, Kim ED, Chen ZJ. Duplicate genes increase expression diversity in closely related species and allopolyploids. PNAS. 2008;106(7):2295-300.

18. Bremer G. Problems in breeding and cytology of sugarcane. Euphytica. 1961;10(3):325-42.

19. Figueira TRES, Okura V, Silva FRD, Silva MJD, Kudrna D, Ammiraju JS, Talag J, Wing R, Arruda P. A BAC library of the SP80-3280 sugarcane variety (saccharum sp.) and its inferred microsynteny with the sorghum genome. BMC Res Notes. 2012;5:185.

20. Theocharis A. Cle'ment C, Barka EA. physiological and molecular changes in plants grown at low temperatures. Planta. 2012;235(6):1091-105.

21. Zhu JK. Abiotic stress signaling and responses in plants. Cell. 2016;167(2): 313-24.

22. Manjunatha T, Chandran K, Mahesh P, Mohanraj L. Appunu. Research article characterization of Saccharum spontaneum accessions from north-eastern India. Electron J Plant Breed. 2018;9(1):38-50.
23. Zhang BQ, Yang LT, Li YRS. Physiological and biochemical characteristics related to cold resistance in sugarcane. Sugar Tech. 2015;17(1):49-58.

24. Yang $Y$, Zhang X, Su YC, Zou JK, Wang ZT, Xu LP, Que YX. miRNA alteration is an important mechanism in sugarcane response to low-temperature environment. BMC Genomics. 2017;18(1):833.

25. Selvarajan D, Mohan CM, Dhandapani V, Nerkar G, Jayanarayanan AN, Mohanan MV, Murugan N, Kaur L, Chennappa M, Kumar R, Meena M, Ram B, Chinnaswamy A. Differential gene expression profiling through transcriptome approach of Saccharum spontaneum L. under low temperature stress reveals genes potentially involved in cold acclimation. 3 Biotech. 2018;8(4):195.

26. Dharshini S, Chakravarthi M, Narayan JA, Manoj VM, Naveenarani M, Kumar R, Meena M, Ram B, Appunu C. De novo sequencing and transcriptome analysis of a low temperature tolerant Saccharum spontaneum clone IND 00-1037. J Biotechnol. 2016:231:280-94.

27. Yu XH, Wang XH, Yang QH. Genetic diversity and phylogenetic relationship of Saccharum spontaneum L. with different Ploidy levels based on SRAP markers. Sugar Tech. 2019;21(5):802-14.

28. Liu XL, Li XJ, Liu HB, Xu CH, Lin XQ, Li CJ, Deng ZH. Phylogenetic analysis of different Ploidy Saccharum spontaneum based on rDNA-ITS sequences. PLoS One. 2016;11(3):e0151524.

29. Carroll AB, Pallardy SG, Galen C. Drought stress, plant water status, and floral trait expression in fireweed, Epilobium angustifolium (Onagraceae). Am J Bot. 2001;88(3):438-46.

30. Nilson SE, Assmann SM. The control of transpiration. Insights from Arabidopsis1. Plant Physiol. 2007;147(1):19-27.

31. Wei CX, Xie PS, Zhou WD, Chen YF, Zhang J, Hui HY. Observation on morphological structure of leaf epidermis of Ophiopogon japonicas, Liriope spicata and Liriope platyphylla. J Plant Resour Environ. 2008;17(4):9-15 (In China).

32. Wang YJ. Statistics analysis on the quantitative characteristics of bulliform cells in wheat leaves. J Triticeae Crops. 2009;29(6):1100-4 (In China).

33. Ogburn RM, Edwards EJ. Quantifying succulence: a rapid, physiologically meaningful metric of plant water storage. Plant Cell Environ. 2012;35(9): 1533-42.

34. Zeng Y, Yu J, Cang J, Liu LJ, Mu YC, Wang JH, Zhang D. Detection of sugar accumulation and expression levels of correlative key enzymes in winter wheat (Triticum aestivum) at low temperatures. Biosci Biotechnol Biochem. 2011;75(4):681-7.

35. Sales CRG, Ribeiro RV, Silveira JAG, Machado EC, Martins MO, Lagôa AMMA. Superoxide dismutase and ascorbate peroxidase improve the recovery of photosynthesis in sugarcane plants subjected to water deficit and low substrate temperature. Plant Physiol Biochem. 2013;73(6):326-36.

36. Oh SJ, Kim YS, Kwon CW, Park HK, Jeong JS, Kim JK. Overexpression of the transcription factor AP37 in Rice improves grain yield under drought Conditions $^{1[\mathrm{~W}][0 \mathrm{~A}]}$. Plant Physiol. 2009;150(3):1368-79.

37. Zhang N, Wang SS, Xf Z, Dong ZD, Chen F, DQS C. Transcriptome analysis of the Chinese bread wheat cultivar Yunong 201 and its ethyl methanesulfonate mutant line. Gene. 2016;575(2):285-93.

38. Zhang L, Lin Q, Feng YZ, Fan XM, Zou F, Yuan DY, Zeng XC, Cao HP. Transcriptomic identification and expression of starch and sucrose metabolism genes in the seeds of Chinese chestnut (Castanea mollissima). J Agric Food Chem. 2015;63(3):929-42.

39. Li P, Yang H, Wang L, Liu HJ, Huo HQ, Zhang CJ, Liu AZ, Zhu AD, Hu JY, Lin YJ, Liu L. Physiological and Transcriptome Analyses Reveal Short-Term Responses and Formation of Memory Under Drought Stress in Rice. Front Genet. 2019;10:55.

40. Paul M, Pellny T, Goddijn D. Enhancing photosynthesis with sugar signals. Trends Plant Sci. 2001;5(6):197-200.

41. Kandror O, DeLeon A, Goldberg AL. Trehalose synthesis is induced upon exposure of Escherichia coli to cold and is essential for viability at low temperatures. PNAS. 2002;99(15):9727-32.

42. Goddijn OJM, Dun KV. Trehalose metabolism in plants. Trends Plant Sci. 1999:4(8):315-9.

43. Alexander AG. Studies on trehalase in Saccharum spp. leaf and storage tissues. Plant Cell Physiol. 1972;14(1):157-68.

44. Elbein AD, Pan YT, Pastuszak I, Carroll D. New insights on trehalose: a multifunctionalmolecule. Glycobiology. 2003;13(4):17-27.

45. Pilon-Smits EAH, Terry N, Sears T, Kim H, Zayed A, Hwang S, Dun KV, Voogo E, Verwoerd TC, Krutwagen RWHH, Goddijn OJM. Trehalose-producing transgenic tobacco plants show improved growth performance under drought stress. J Plant Physiol. 1998;152:525-32. 
46. Garg AK, Kim JK, Owens TG, Ranwala AP, Choi YD, Kochian LV, Wu RJ. Trehalose accumulation in rice plants confers high tolerance levels to different abiotic stresses. PNAS. 2002;99(25):15898-903.

47. Chinnusamy V, Zhu JH, Zhu JK. Cold stress regulation of gene expression in plants. Trends Plant Sci. 2007;12(10):444-51.

48. Qiu JH, Hou YX, Wang YF, Li ZY, Zhao J, Tong XH, Lin HY, Wei XJ, Ao HJ, Zhang J. A Comprehensive Proteomic Survey of ABA-Induced Protein Phosphorylation in Rice (Oryza sativa L.). Int J Mol Sci. 2017;18(1):e60.

49. Kinoshita T, Delgado AC, Seto H, Hiranuma S, Fujioka S, Yoshida S, Chory J. Binding of brassinosteroids to the extracellular domain of plant receptor kinase BRI1. Nature. 2005;433(7022):167-71.

50. Eremina M, Unterholzner SJ, Rathnayake A, Castellanos M, Khan M, Kugler KG, May ST, Mayer KF, Rozhon W, Poppenberger B. Brassinosteroids participate in the control of basal and acquired freezing tolerance of plants. PANS. 2016:40(113):5982-91.

51. Wu XX, He J, Zhu ZW, Yang SJ, Zha DS. Protection of photosynthesis and antioxidative system by 24-epibrassinolide in Solanum melongena under cold stress. Biol Plant. 2014;58(1):185-8.

52. Li BQ, Zhang CF, Cao BH, Qin GZ, Wang WH, Tian SP. Brassinolide enhances cold stress tolerance of fruit by regulating plasma membrane proteins and lipids. Amino Acids. 2012;43(6):2469-80.

53. Anuradha S, Rao SDR. Effect of brassinosteroids on salinity stress induced inhibition of seed germination and seedling growth of rice (Oryza sativa L.). Plant Growth Regul. 2001;33(2):151-3.

54. Sreeramulu S, Mostizky Y, Sunitha S, Shani E, Nahum H, Salomon D, Hayun LB, Gruetter C, Rauh D, Ori N, Sessa G. BSKs are partially redundant positive regulators of brassinosteroid signaling in Arabidopsis. Plant J. 2013;74(6): 905-19.

55. Li ZY, XU ZS, He GY, Yang GX, Chen M, Li LC, Ma YZ. A mutation in Arabidopsis BSK5 encoding a brassinosteroid-signaling kinase protein affects responses to salinity and abscisic acid. Biochem Biophys Res Commun. 2012:426(4):522-7.

56. Peer YVD, Mizrachi E, Marchal K. The evolutionary significance of polyploidy. Nat Rev Genet. 2017;18(7):411-24.

57. Jackson PA. Breeding for improved sugar content in sugarcane. Field Crop Res. 2005:92(2-3):277-90.

58. Yang S, Li XT, Huang F, Huang YJ, Liu XL, Wu JY, Wang QN, Deng ZH, Chen RK, Zhang MQ. A new method based on SNP of nrDNA-ITS to identify Saccharum spontaneum and ITS progeny in the genus Saccharum. PLoS One. 2018;13(5):e0197458.

59. NY/T 1488-2007. Technical code for evaluating germ plasm resourcesSugarcane (Saccharum L.) [S]. Beijing, China Agriculture Press, 2007. (In China).

60. Huang X, Chen MH, Yang LT, Li YR, Wu JM. Effects of exogenous Abscisic acid on cell membrane and endogenous hormone contents in leaves of sugarcane seedlings under cold stress. Sugar Tech. 2015;17(1):59-64.

61. Cia MC, Guimaraes ACR, Medici LO, Chabregas SM, Azevedo RA. Antioxidant responses to water deficit by drought-tolerant and -sensitive sugarcane varieties. Ann Appl Biol. 2012;161:313-24.

62. Grabherr MG, Haas BJ, Yassour M, Levin JH, Thompson DA, Amit I, Adiconis $X$, Fan L, Raychowdhury R, Zeng QD, et al. Trinity: reconstructing a fulllength transcriptome without a genome from RNA-Seq data. Nat Biotechnol. 2013;29(7):644-52.

63. Li B, Dewey CN. RSEM: accurate transcript quantification from RNA-Seq data with or without a reference genome. BMC Bioinformatics. 2011:12:323.

64. Li CN, Srivastava MK, Nong Q, Yang LT, Li YR. Molecular cloning and characterization of SoNCED, a novel gene encoding 9-cis-epoxycarotenoid dioxygenase from sugarcane (Saccharum officinarum L.). Genes Genom. 2013;35(1):101-9.

65. Yang YT, Zhang X, Chen Y, Guo JL, Ling H, Gao SW, Su YC, Que YX, Xu LP. Selection of reference genes for normalization of MicroRNA expression by RT-qPCR in sugarcane buds under cold stress. Front Plant Sci. 2016;7:86.

66. Jain M, Nijhawan A, Tyagi AK, Khurana JP. Validation of housekeeping genes as internal control for studying gene expression in rice by quantitative realtime PCR. Biochem Biophys Res Commun. 2006;345(2):646-51.

67. Livak KJ, Schmittgen TD. Analysis of relative gene expression data using RealTime quantitative PCR and the $2^{-\Delta \Delta C t}$ method. Methods. 2001;25(4):402-8

68. Pallant J. SPSS survival manual: a step by step guide to data analysis using IBM SPSS. Aust N Z J Public Health. 2013;37(6):597-8.

69. Kornbrot D. Statistical software for microcomputers: SigmaPlot 2000 and SigmaStat2. Br J Math Stat Psychol. 2000;53(2):335-7.
70. Deng WK, Wang YB, Liu ZX, Cheng H, Xue Y. Heml: a toolkit for illustrating Heatmaps. PLoS One. 2014:11(9):e111988.

\section{Publisher's Note}

Springer Nature remains neutral with regard to jurisdictional claims in published maps and institutional affiliations.
Ready to submit your research? Choose BMC and benefit from:

- fast, convenient online submission

- thorough peer review by experienced researchers in your field

- rapid publication on acceptance

- support for research data, including large and complex data types

- gold Open Access which fosters wider collaboration and increased citations

- maximum visibility for your research: over $100 \mathrm{M}$ website views per year

At $\mathrm{BMC}$, research is always in progress.

Learn more biomedcentral.com/submissions 